NBER WORKING PAPER SERIES

\title{
ESTIMATING RICARDIAN MODELS WITH PANEL DATA
}

\author{
Emanuele Massetti \\ Robert Mendelsohn \\ Working Paper 17101 \\ http://www.nber.org/papers/w17101
NATIONAL BUREAU OF ECONOMIC RESEARCH
1050 Massachusetts Avenue
Cambridge, MA 02138
June 2011

We are grateful to William Nordhaus for comments on an earlier draft of this paper. Emanuele Massetti gratefully acknowledges financial support from the EU sponsored PASHMINA project and by the research project D.1 - 2010, Metodi e modelli matematici per le decisioni nelle scienze sociali, Catholic University of Milan. The views expressed herein are those of the authors and do not necessarily reflect the views of the National Bureau of Economic Research.

NBER working papers are circulated for discussion and comment purposes. They have not been peerreviewed or been subject to the review by the NBER Board of Directors that accompanies official NBER publications.

(C) 2011 by Emanuele Massetti and Robert Mendelsohn. All rights reserved. Short sections of text, not to exceed two paragraphs, may be quoted without explicit permission provided that full credit, including (c) notice, is given to the source. 
Estimating Ricardian Models With Panel Data

Emanuele Massetti and Robert Mendelsohn

NBER Working Paper No. 17101

June 2011

JEL No. Q1,Q12,Q51,Q54

\title{
$\underline{\text { ABSTRACT }}$
}

Many nonmarket valuation models, such as the Ricardian model, have been estimated using cross sectional methods with a single year of data. Although multiple years of data should increase the robustness of such methods, repeated cross sections suggest the results are not stable. We argue that repeated cross sections do not properly specify the model. Panel methods that correctly specify the Ricardian model are stable over time. The results suggest that many cross sectional methods including hedonic studies and travel cost studies could be enhanced using panel data.

\author{
Emanuele Massetti \\ Fondazione Eni Enrico Mattei \\ C.so Magenta, 63 \\ 20123 Milano - Italy \\ and Centro Euro-Mediterraneo per i Cambiamenti Climatici \\ emanuele.massetti@feem.it \\ Robert Mendelsohn \\ School of Forestry \& Environmental Studies \\ Yale University \\ 195 Prospect St. \\ New Haven, CT 06511 \\ robert.mendelsohn@yale.edu
}




\section{Introduction}

The traditional Ricardian model is a cross sectional analysis that measures the long term impacts of climate change on agriculture (Mendelsohn, Nordhaus, and Shaw 1994) (MNS). The technique has been applied successfully in over 27 countries across the world (Mendelsohn and Dinar 2009). However, the technique has almost always been applied to just a single year of data (Sanghi and Mendelsohn 2008; Mendelsohn, Dinar and Sanghi 2001 are exceptions). Applying the method to repeated cross sections reveals that these single year studies do not lead to stable results over time (Deschenes and Greenstone 2007) (DG). Instead of cross sectional analysis, DG argue that one should focus on the intertemporal variation in weather to detect climate change effects.

We argue that the intertemporal variation is simply not as useful as the cross sectional variation for measuring climate change effects. The purpose of these studies is to measure the impacts of climate change (changes in long term weather) on agriculture. Interannual changes in weather are a poor proxy for climate. Weather changes are surprises to farmers. Farmers only have limited opportunities to adapt to weather. In contrast, climate is long run weather. Farmers can do a great deal to adapt to changes in climate. For example, they can adjust which crops to plant (Kurukulasuriya and Mendelsohn 2008; Seo and Mendelsohn 2008a) and which livestock to raise (Seo and Mendelsohn 2008b). Studying the impact of weather cannot capture any of these adjustments to climate. The intertemporal analysis of weather may provide an accurate measurement but of the wrong phenomenon.

Further, we argue that if panel data is available for the Ricardian method, repeated cross sections are the wrong way to estimate the consequences. Instead, one should use panel data 
methods that properly specify which coefficients should vary over time and which should remain stable. Correct specification applies to far more than just the Ricardian model. There are many cross sectional methods that have been developed to measure the value of the environment. Hedonic property value studies have been used to value natural resource amenities such as waterfront (Parsons and $\mathrm{Wu}$ 1991) or proximity to open space and disamenities such as hazardous waste sites and airport noise (see review by Palmquist and Smith 2002). Hedonic wage studies have measured the value of risk as a factor in wages (see reviews by Mrozek and Taylor 2002 and Viscusi and Aldy 2003). Travel cost studies have measured the recreational value of natural resources such as parks, coral reefs, and forests (see review by Mendelsohn and Olmstead 2009).

DG use a series of independent cross sectional regressions to estimate a set of Ricardian models. They find that, at least for this application of the Ricardian method, the results across the regressions are not stable. They raise doubts about using cross sectional valuation methods at all. However, there are some problems with the DG analysis. We revise the original DG analysis to correct for climate data problems, missing observations, omitted variables, and functional form. We then re-examine the results from repeated cross sections.

We compare the results or the repeated cross section with the results from panel methods. We argue that repeated cross sections are mis-specified. If carefully specified, the results are robust. We present two panel data approaches, a two stage model by Cheng Hsiao (2008) and a single stage "pooled" model. Using US data from the same Census time periods as the DG study, we show that both panel data approaches yield stable results. The results imply that panel methods would likely work not only for Ricardian studies but also for other cross sectional models such as hedonic and travel cost studies. 


\section{Methodology}

The Ricardian method assumes the value of farmland $(\mathrm{V})$ is equal to the present value of net revenue from farm related activities (MNS, 1994). Land values are therefore equal to:

$V=\int\left[\sum P Q(I, C, G, S)-R^{\prime} I\right] e^{-\delta t} d t$,

where $V$ is the value of farmland per hectare, $\boldsymbol{P}$ is the market price of output, $\boldsymbol{Q}$ is output, $\boldsymbol{I}$ is a vector of purchased inputs (other than land), $\boldsymbol{C}$ is a vector of climate variables, $\boldsymbol{G}$ is a vector of geographic variables, $\boldsymbol{S}$ is a vector of soil variables, $\mathbf{R}$ is a vector of input prices, $t$ is time and $\delta$ is the discount rate. Farmers are assumed to maximize net revenues by choosing $\mathbf{I}$, given climate, soil, geographic variables, market prices, and other exogenous socio-economic conditions.

Solving (1) to maximize net revenue leads to a reduced form model where $V$ is strictly a function of the exogenous variables facing a farmer: $\boldsymbol{S}, \boldsymbol{G}, \boldsymbol{C}, \boldsymbol{R}, \boldsymbol{r}$, and $\boldsymbol{Z}$. In this paper, we focus on applying the Ricardian model to a panel data set. For expositional purposes, we group these variables into a vector of time varying variables, $\boldsymbol{X}$, a vector of time invariant control variables $\boldsymbol{Z}$, and a vector of climate variables $\boldsymbol{C}$. The time varying variables include population density, income, subsidies, and residential housing prices. The time invariant variables (at least over the panel) include soil and geographic variables. The Ricardian model has the general form:

$V=f(\mathbf{X}, \mathbf{Z}, C)$.

Traditionally, the Ricardian model is estimated across a single cross section:

$V_{i}=X_{i} \boldsymbol{\beta}+Z_{i} \gamma+C_{i} \varphi+u_{i}$,

where $i$ varies across space. The relationship between the climate variables and land value is assumed to be quadratic so that the climate vector includes squared terms. Because the effect of 
climate on land value varies across seasons (MNS 1994), temperature and precipitation are introduced for each of four seasons. The estimated coefficients in the model are $\beta$, $\gamma$, and $\varphi$. Following DG, we control for heteroscedasticity with Weighted Least Squares (WLS). As with DG, we use farmland by county in each year for a weight. Following Schlenker, Hanemann, and Fisher (2005), we also correct for spatial correlation following the non-parametric approach of Conley (1999).

DG estimate the Ricardian model using repeated independent cross sections. They estimate the following model with panel data:

$V_{i, t}=X_{i, t}^{\prime} \boldsymbol{\beta}_{t}+Z^{\prime}{ }_{i} \gamma_{t}+C^{\prime}{ }_{i} \varphi_{t}+u_{i, t}$,

where $X_{i . t}^{\prime}$ is now a matrix of time varying control variables. The estimated coefficients are all matrices. That is, the coefficients are all allowed to vary over time: $\beta_{t}, \gamma_{t}$, and $\varphi_{t}$. DG argue that the resulting Ricardian model is nonsensical because $\varphi_{k} \neq \varphi_{l}$ over time in their regressions.

However, we argue that repeated cross sections are effectively mis-specified because the coefficients of the time varying variables should not change over time. In an ideal panel data model, the coefficients of the time invariant variables should also not change. The correctly specified Ricardian model is:

$V_{i, t}=X_{i, t}^{\prime} \boldsymbol{\beta}+Z_{i}^{\prime} \gamma+C_{i}^{\prime} \varphi+u_{i, t}$,

where $\boldsymbol{\beta}, \boldsymbol{\gamma}$, and $\boldsymbol{\varphi}$ are time invariant vectors. By allowing $\boldsymbol{\beta}$ and $\boldsymbol{\gamma}$ to vary over time, the repeated cross section causes $\varphi$ to also vary. 
We explore two ways to estimate the Ricardian model with panel data. One way is to pool the entire data set and estimate the model above in a single stage using Equation (5). The second approach is to estimate two stages (Cheng Hsiao 2008). In the first stage, land value is regressed on the time varying variables using the covariance method with county fixed effects:

$V_{i, t}=X_{i, t}^{\prime} \boldsymbol{\beta}+\mathbf{e} \alpha_{i}+\varepsilon_{i, t}$,

where $\mathbf{e}$ is a vector of county fixed effects (dummies), and $\varepsilon_{i, t}$ is the resulting error term. By including county fixed effects, the first stage in the Hsiao model does a better job of controlling for omitted variables that are correlated with space. In the second stage, the time-mean residuals are regressed on the time invariant variables using WLS: ${ }^{1}$

$\overline{V_{i}}-\bar{X}_{i}^{\prime} \hat{\boldsymbol{\beta}}_{C V}=\mathbf{e} \alpha_{i}+\overline{\varepsilon_{i}}=Z_{i}^{\prime} \boldsymbol{\gamma}+C_{i}^{\prime} \varphi+\bar{u}_{i}$

In order to test whether the climate coefficients are stable over time, we estimate a variant of both panel models. For both the Hsiao model and the pooled model, we allow the climate coefficients to change over time. In the Hsiao model, the first stage of the model remains the same. However, in the second stage, we estimate a separate set of coefficients, $\varphi_{t}$, for each year:

$V_{i, t}-X_{i, t}^{\prime} \hat{\boldsymbol{\beta}}_{C V}=Z_{i}^{\prime} \gamma+C_{i}^{\prime} \varphi_{t}+u_{i, t}$

where the coefficients associated with climate are allowed to vary over time. The model is equivalent to creating a set of time dummies for each year and interacting these time dummies

${ }^{1}$ With $\bar{V}_{i}=\frac{1}{T} \sum_{t} \bar{V}_{i, t}$ and $X_{i}=\frac{1}{T} \sum_{t} X_{i, t}$. 
with the climate variables. Instead of a single set of coefficients for the climate variables, one has a different estimate for each year.

With the "pooled" model, we interact the climate variables with year dummies. They are then combined with the time varying variables in a single regression on land value:

$$
V_{i, t}=X_{i, t}^{\prime} \boldsymbol{\beta}+Z^{\prime}{ }_{i} \gamma+C_{i}^{\prime} \varphi_{t}+u_{i, t}
$$

This model yields a set of coefficients for all the climate variables for each year. One can then test whether the coefficients are stable over time.

The welfare impact $W$ of climate change on US agriculture is obtained by computing the difference between the value of farmland under the new climate and the value of farmland under the current climate. We use the estimated coefficients, the average farmland in each county, and the predicted change in climate from $\mathrm{C}_{0}$ to $\mathrm{C}_{1}$ :

$$
W_{t}=\sum_{i}\left[V_{i, t}\left(C_{1}\right)-V_{i, t}\left(C_{0}\right)\right] F_{i}
$$

Of course, the best forecast of welfare effects in the future should rely on the expected value of farmland in the future not the past. This would require modeling not only how farmland is expected to change over time from technological and economic forces but also how it might change in response to climate change (Mendelsohn, Nordhaus, and Shaw 1996). In the present analysis, we employ a less sophisticated approach and we use as expected value of farmland the average number of acres in farmland over the observed Census years.

\section{Data}

Following DG, we have constructed a balanced panel with observations from the contiguous 48 States over the U.S. Agricultural Census years of 1978, 1982, 1987, 1992, 1997 
and 2002. Units of measurement follow the metric system; economic variables have all been converted to constant 2000 USD using the GDP deflator. There are many counties missing in the DG analysis. The DG analysis has only 2124 counties of the 3048 counties in the lower 48 states that have agriculture. We are able to include 2914. Our analysis is based on $97 \%$ of US farmland whereas the DG analysis is based on only $72 \%$. The omission of so many counties has implications for both the estimation of the regressions and the calculation of welfare effects. ${ }^{2}$

Because there are data errors in the DG climate variables (see Fisher et al 2010), we rely on the 1971-2000 monthly precipitations and mean temperature normals computed by the National Climatic Data Center for 7,467 weather stations in the contiguous 48 States. The standard definition of a climate normal is the average weather over a 30 year period. Following MNS, we estimate a quadratic climate surface as a function of longitude, latitude, elevation and distance from coastline, for the contiguous 48 States. For each county of origin, we calculate the weather surface using the weather stations within 500 miles. The data is weighted to give nearby stations more weight. The predicted values from that surface are used to yield monthly climate variables for each county. The seasonal climate is the arithmetic average of climate variables in winter (December, January, February), spring (March, April, May), summer (June, July, August) and autumn (September, October, November).

For farmland value, land in farms, and other agricultural data, we rely on the U.S. Census of Agriculture (USDA 1978, 1982, 1987, 1992, 1997, 2002). The dependent variable in our panel is the average estimated value of land and buildings per hectare of land in farm.

\footnotetext{
${ }^{2}$ It is not clear why DG omit so many counties from their analysis since data is available for most of them.
} 
One point stressed by DG is that it is important to include missing variables. We include several variables not used in DG or MNS. We measure the long-term availability of water to farmers using the average fresh surface water withdrawals by county over time (United States Geological Survey, 1988, 1993, 1998, 2004). Because water rights are based on use in the U.S., average withdrawals capture underlying water rights and can be largely considered as exogenous (Mendelsohn and Dinar 2003). Data about greenhouses by county are included from the Census of Agriculture. The analysis also captures subsidies as measured by the Census of Agriculture. In order to control for local land markets, we use the median value of owner-occupied housing in each county as a proxy for the opportunity cost of keeping land in farms. These data are available at the county level from the US Census of Population and Housing every ten years. We extrapolate house values using linear trends between Census years.

\section{Results}

In the Appendix, we present the regression results of estimating the repeat cross sections across the six years of data (Table A1). Note that all the coefficients are permitted to vary and that they are in general not stable. Of particular importance is the instability of the climate coefficients. Even correcting for functional form, missing observations, errors in climate variables, and omitted variables, the climate coefficients vary over time. This can be readily seen by calculating the marginal effects of temperature and precipitation in Table 1. The seasonal and annual marginals vary a great deal of over time. The seasonal temperature marginals are quite significant but they are offsetting so that the annual temperature marginals are not significant. In contrast, both the seasonal and annual precipitation marginals are significant. 
Table 1-Temperature and precipitations marginals at the average U.S. climate. Annual and seasonal effects. Enhanced cross section method.

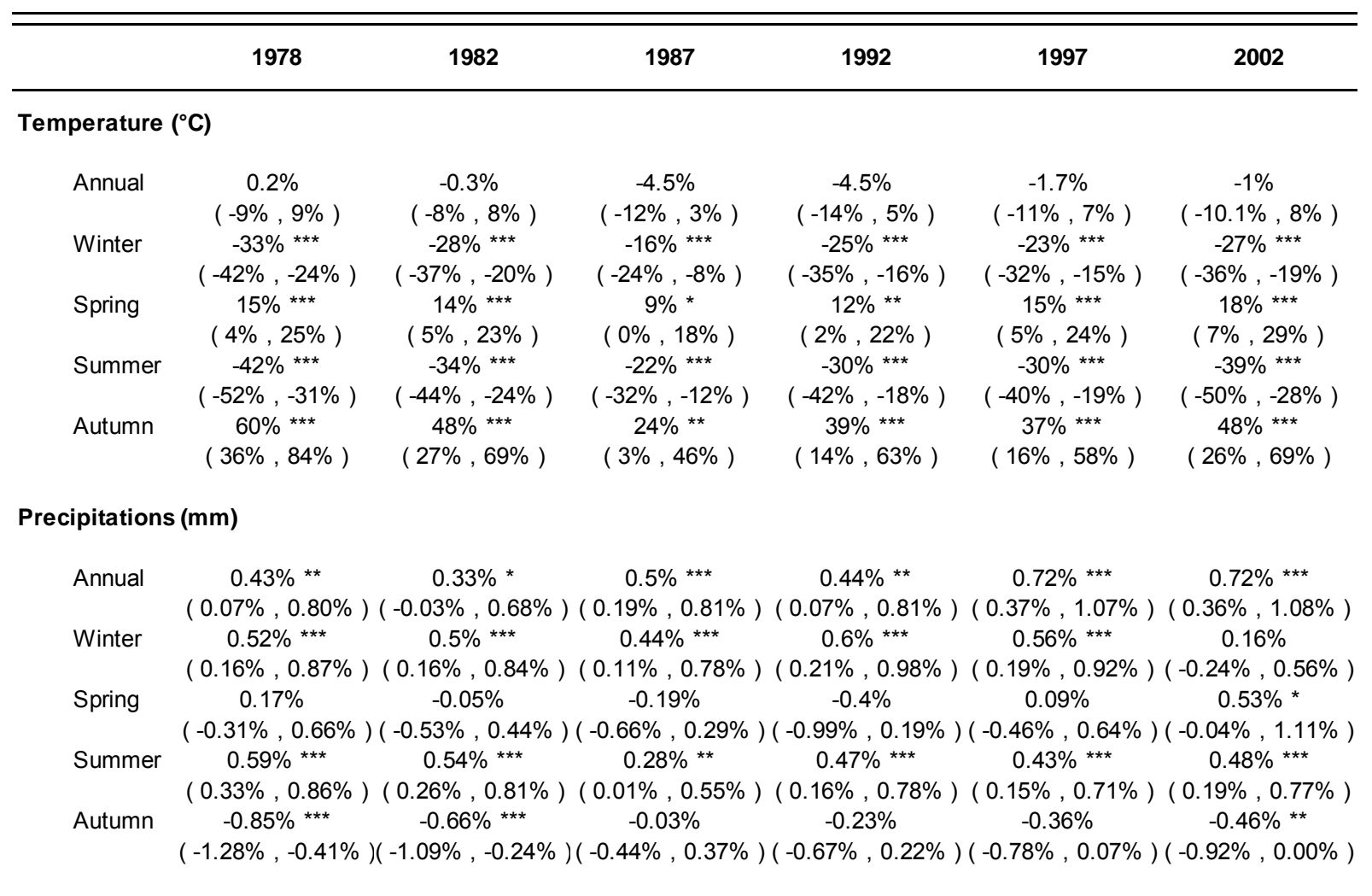

Notes: \% of land value per hectare of farmland. Standard errors corrected for spatial correlation, cutoff point at 3 degrees. $* \mathrm{p}<0.01 ; * * \mathrm{p}<0.05$; ${ }^{* * *} \mathrm{p}<0.001 .95 \%$ confidence intervals are in parenthesis. Average temperature $\left({ }^{\circ} \mathrm{C}\right)$ in Winter: 0.8 , Spring: 11.8 , Summer: 22.9 , Autumn: 13.0 and average precipitations (mm) in Winter: 68, Spring: 88, Summer: 92, Autumn: 77.

The range of climate coefficients across time can also be seen by calculating the welfare impacts of a uniform temperature increase of $2.7^{\circ} \mathrm{C}$ with an 8 percent increase in precipitation (the scenario used in MNS and DG). Using this same climate change but the different climate coefficients estimated in each year, Figure 1 demonstrates that the welfare results from this uniform change are not the same across years with the repeated cross sections. 
Figure 1-The welfare impacts of a uniform temperature increase of $2.7^{\circ} \mathrm{C}$ and an 8 percent increase in precipitation in the enhanced repeated cross section.

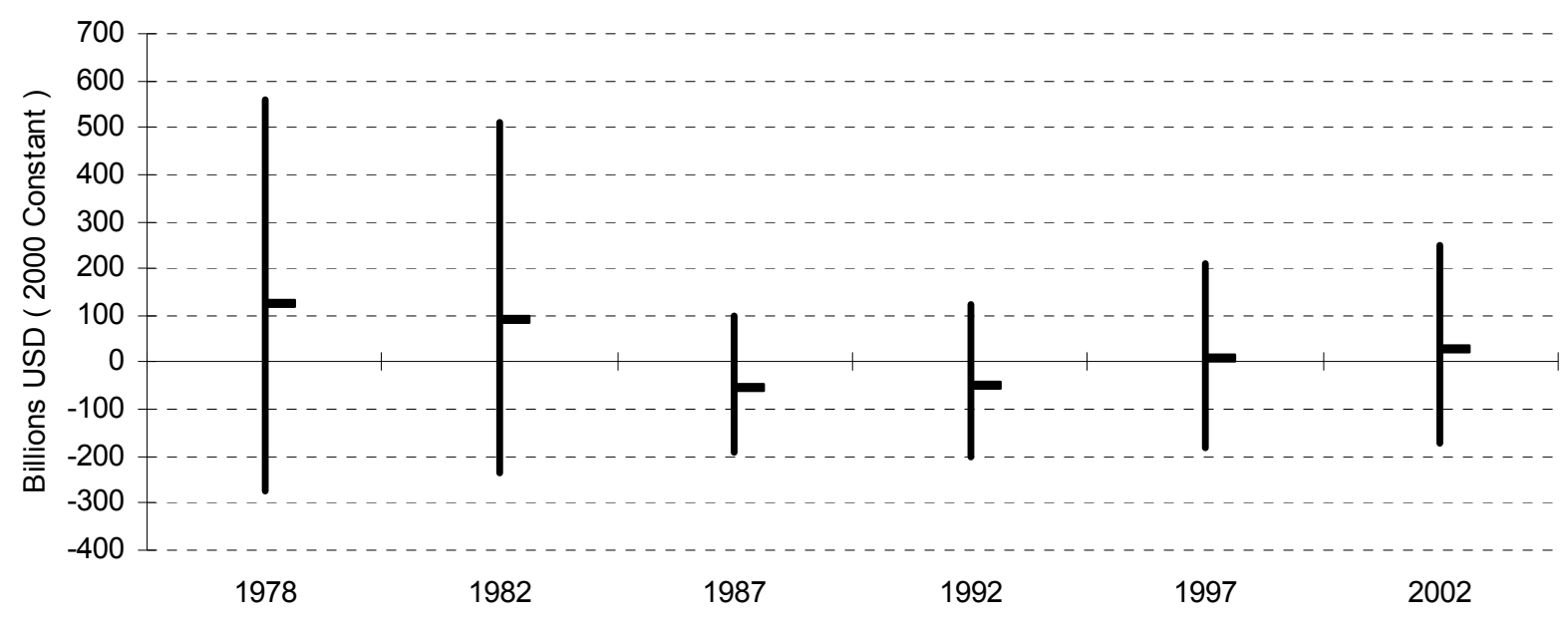

Notes: Central values and 95 percent bootstrap confidence intervals based on 1,000 repetitions.

Using the same data set, functional form, and variables that are used in the repeated cross sections, we estimate the Hsiao and pooled models. The first stage of the Hsiao model includes income, density, density squared, greenhouses, subsidies, and residential housing values, time dummies for each year, and county fixed effects (which are not reported). There is a single coefficient for each time varying variable. The second stage includes geographic, soils, and climate variables. The pooled model has the exact same independent variables except that county fixed effects are not utilized.

For both the Hsiao and pooled models, Table 2A presents the coefficients of the time varying variables and Table $2 \mathrm{~B}$ presents the coefficients of the time invariant variables. In Table $2 \mathrm{~A}$ the $\mathrm{R}^{2}$ values for the Hsiao model are high because they include county fixed effects that are not shown. Comparing the coefficients of the time varying variables in the Hsiao and pooled models, income, density and density squared are significantly different. In the pooled model, 
Table 2A-Coefficients of time varying variables in the Hsiao and pooled model

\begin{tabular}{lccccc}
\hline \hline Model & Hsiao & Pooled Model & & Hsiao & Pooled Model \\
\hline Income & 0.00538 & 0.00914 & 1982 dummy & -0.0366 & -0.0309 \\
& $(3.5)$ & $(4.71)$ & & $(-4.51)$ & $(-1.87)$ \\
Density & 0.00466 & 0.00184 & 1987 dummy & -0.351 & -0.477 \\
& $(6.08)$ & $(9.21)$ & & $(-29.99)$ & $(-22.6)$ \\
Density sq & $-3.45 \mathrm{E}-06$ & $-1.21 \mathrm{E}-06$ & 1992 dummy & -0.468 & -0.511 \\
& $(-5.25)$ & $(-5.95)$ & & $(-43.05)$ & $(-26.7)$ \\
Share of greenhouses & 0.291 & 0.331 & 1997 dummy & -0.400 & -0.424 \\
& $(1.86)$ & $(2.58)$ & & $(-33.05)$ & $(-21.5)$ \\
Government transfers & -0.00105 & 0.00178 & 2002 dummy & -0.344 & -0.400 \\
& $(-9.49)$ & $(10.2)$ & & $(-23.17)$ & $(-18.4)$ \\
House price index & 0.00614 & 0.00601 & Constant & 7.20 & - \\
& $(18.2)$ & $(17.8)$ & Adjusted R-Squared & 0.80 & - \\
\hline
\end{tabular}

Notes: t-statistics in parenthesis. County fixed effects not shown.

income has a much larger positive effect but density and density squared have a much smaller effect. The coefficients of the remaining time varying variables in the two regressions are quite similar.

The coefficients of the time invariant variables in the Hsiao and pooled models in Table 2B are not significantly different. The major difference between the two models is that the t-statistics of the pooled model are higher because the pooled model assumes there are more observations. The true number of independent observations lies between the two methods and depends upon whether there are independent errors because of random intertemporal fluctuations suggesting more observations or whether there are consistent errors across time suggesting fewer observations. 
Table 2B-Coefficients of time invariant variables in the Hsiao and pooled Model

\begin{tabular}{|c|c|c|c|c|c|}
\hline Model & Hsiao & Pooled Model & & Hsiao & Pooled Model \\
\hline Temp Winter & $\begin{array}{l}-0.259 \\
(-5.43)\end{array}$ & $\begin{array}{l}-0.260 \\
(-5.96)\end{array}$ & Salinity & $\begin{array}{l}-0.093 \\
(-0.9)\end{array}$ & $\begin{array}{l}-0.102 \\
(-1.12)\end{array}$ \\
\hline Temp Winter sq & $\begin{array}{c}-3.08 \mathrm{E}-03 \\
(-1.47)\end{array}$ & $\begin{array}{c}-4.18 \mathrm{E}-03 \\
(-2.28)\end{array}$ & Flooding & $\begin{array}{l}-0.238 \\
(-2.24)\end{array}$ & $\begin{array}{c}-0.254 \\
(-2.36)\end{array}$ \\
\hline Temp Spring & $\begin{array}{c}0.374 \\
(2.99)\end{array}$ & $\begin{array}{c}0.359 \\
(3.61)\end{array}$ & Wet index & $\begin{array}{c}0.225 \\
(2.07)\end{array}$ & $\begin{array}{c}0.154 \\
(1.66)\end{array}$ \\
\hline Temp Spring sq & $\begin{array}{c}-6.93 \mathrm{E}-03 \\
(-1.39)\end{array}$ & $\begin{array}{c}-8.00 \mathrm{E}-03 \\
(-1.88)\end{array}$ & K-factor & $\begin{array}{l}-1.00 \\
(-2.16)\end{array}$ & $\begin{array}{c}-1.04 \\
(-2.97)\end{array}$ \\
\hline Temp Summer & $\begin{array}{c}-0.752 \\
(-3.34)\end{array}$ & $\begin{array}{c}-0.661 \\
(-3.66)\end{array}$ & Length of slope & $\begin{array}{c}1.20 \mathrm{E}-04 \\
(1.15)\end{array}$ & $\begin{array}{c}9.45 \mathrm{E}-05 \\
(0.96)\end{array}$ \\
\hline Temp Summer sq & $\begin{array}{c}9.56 \mathrm{E}-03 \\
(2.19)\end{array}$ & $\begin{array}{c}7.18 \mathrm{E}-03 \\
(1.97)\end{array}$ & Sand & $\begin{array}{c}0.241 \\
(1.28)\end{array}$ & $\begin{array}{c}0.111 \\
(0.76)\end{array}$ \\
\hline Temp Autumn & $\begin{array}{c}0.259 \\
(0.95)\end{array}$ & $\begin{array}{c}0.215 \\
(0.99)\end{array}$ & Clay & $\begin{array}{l}-0.106 \\
(-1.2)\end{array}$ & $\begin{array}{c}-0.109 \\
(-1.23)\end{array}$ \\
\hline Temp Autumn sq & $\begin{array}{c}3.60 \mathrm{E}-03 \\
(0.43)\end{array}$ & $\begin{array}{c}7.62 \mathrm{E}-03 \\
(1.13)\end{array}$ & Moisture Level & $\begin{array}{c}1.270 \\
(1.28)\end{array}$ & $\begin{array}{c}0.789 \\
(0.94)\end{array}$ \\
\hline Prec Winter & $\begin{array}{c}0.00708 \\
(2.56)\end{array}$ & $\begin{array}{r}0.00737 \\
(2.92)\end{array}$ & Permeability & $\begin{array}{l}-0.0516 \\
(-1.91)\end{array}$ & $\begin{array}{l}-0.0484 \\
(-1.91)\end{array}$ \\
\hline Prec Winter sq & $\begin{array}{c}-1.81 \mathrm{E}-05 \\
(-1.69)\end{array}$ & $\begin{array}{c}-1.97 \mathrm{E}-05 \\
(-2.08)\end{array}$ & Latitude & $\begin{array}{c}-8.04 \mathrm{E}-03 \\
(-0.24)\end{array}$ & $\begin{array}{c}-4.88 \mathrm{E}-03 \\
(-0.14)\end{array}$ \\
\hline Prec Spring & $\begin{array}{l}0.0178 \\
(4.38)\end{array}$ & $\begin{array}{l}0.0154 \\
(3.31)\end{array}$ & Elevation & $\begin{array}{c}-4.37 \mathrm{E}-04 \\
(-1.97)\end{array}$ & $\begin{array}{c}-4.58 \mathrm{E}-04 \\
(-2)\end{array}$ \\
\hline Prec Spring sq & $\begin{array}{c}-8.16 \mathrm{E}-05 \\
(-3.64)\end{array}$ & $\begin{array}{c}-7.67 \mathrm{E}-05 \\
(-3.08)\end{array}$ & Distance metro areas & $\begin{array}{c}-7.52 \mathrm{E}-04 \\
(-4.07)\end{array}$ & $\begin{array}{c}-8.12 \mathrm{E}-04 \\
(-4.64)\end{array}$ \\
\hline Prec Summer & $\begin{array}{c}0.00453 \\
(1.03)\end{array}$ & $\begin{array}{c}0.00490 \\
(1.19)\end{array}$ & Surface water use & $\begin{array}{l}0.0727 \\
(8.26)\end{array}$ & $\begin{array}{l}0.0738 \\
(8.42)\end{array}$ \\
\hline Prec Summer sq & $\begin{array}{c}1.18 \mathrm{E}-06 \\
(0.05)\end{array}$ & $\begin{array}{c}-6.80 \mathrm{E}-07 \\
(-0.03)\end{array}$ & Constant & $\begin{array}{c}5.62 \\
(2.18)\end{array}$ & $\begin{array}{c}12.2 \\
(4.19)\end{array}$ \\
\hline Prec Autumn & $\begin{array}{l}-0.0208 \\
(-3.07)\end{array}$ & $\begin{array}{l}-0.0203 \\
(-3.36)\end{array}$ & & & \\
\hline Prec Autumn sq & $\begin{array}{c}8.75 \mathrm{E}-05 \\
(2.48)\end{array}$ & $\begin{array}{c}9.26 \mathrm{E}-05 \\
(2.86)\end{array}$ & Adjusted R-squared & 0.80 & 0.84 \\
\hline
\end{tabular}

Notes: t-statistics in parenthesis corrected for spatial correlation, cutoff point at 3 degrees.

Table 3 examines the marginal temperature and precipitation effects of the two panel estimates. Note that the seasonal marginals for both temperature and precipitation are highly significant. However, the annual marginal temperature is not significant. The seasonal effects are offsetting leading to a small annual consequence. The marginal effect of annual precipitation is significant and positive in both models. 
Table 3-Annual and seasonal marginal impact of climate change on U.S. agriculture.

\begin{tabular}{|c|c|c|c|c|c|}
\hline \multicolumn{4}{|c|}{ Temperature $\left({ }^{\circ} \mathrm{C}\right)$} & \multicolumn{2}{|c|}{ Precipitations ( $\mathrm{mm}$ ) } \\
\hline & Hsiao & Pooled & & Hsiao & Pooled \\
\hline Annual & $\begin{array}{c}-1.4 \% \\
(-9.7 \%, 6.8 \%)\end{array}$ & $\begin{array}{c}-1.4 \% \\
(-9.7 \%, 7 \%)\end{array}$ & Annual & $\begin{array}{c}0.55 \%{ }^{* * *} \\
(0.20 \%, 0.90 \%)\end{array}$ & $\begin{array}{c}0.55 \% \text { *** } \\
(0.22 \%, 0.88 \%)\end{array}$ \\
\hline Winter & $\begin{array}{c}-26.4 \%{ }^{* \star \star} \\
(-35.5 \%,-17.3 \%)\end{array}$ & $\begin{array}{c}-26.6 \%{ }^{* * *} \\
(-35.3 \%,-18 \%)\end{array}$ & Winter & $\begin{array}{c}0.46 \%{ }^{* * *} \\
(0.13 \%, 0.79 \%)\end{array}$ & $\begin{array}{c}0.47 \% \%^{* * *} \\
(0.14 \%, 0.80 \%)\end{array}$ \\
\hline Spring & $\begin{array}{c}21.1 \%{ }^{* * *} \\
(11.3 \%, 30.8 \%)\end{array}$ & $\begin{array}{c}17.1 \%{ }^{* * *} \\
(7.5 \%, 26.7 \%)\end{array}$ & Spring & $\begin{array}{c}0.35 \% \\
(-0.15 \%, 0.84 \%)\end{array}$ & $\begin{array}{c}0.20 \% \\
(-0.28 \%, 0.67 \%)\end{array}$ \\
\hline Summer & $\begin{array}{c}-31.4 \%{ }^{* * *} \\
(-43.6 \%,-19.1 \%)\end{array}$ & $\begin{array}{c}-33.1 \%{ }^{* * *} \\
(-43.5 \%,-22.7 \%)\end{array}$ & Summer & $\begin{array}{c}0.47 \%{ }^{* * *} \\
(0.19 \%, 0.76 \%)\end{array}$ & $\begin{array}{c}0.48 \%{ }^{* * *} \\
(0.22 \%, 0.74 \%)\end{array}$ \\
\hline Autumn & $\begin{array}{c}35.3 \%{ }^{* * *} \\
(12.4 \%, 58.1 \%)\end{array}$ & $\begin{array}{c}41.4 \%{ }^{* * *} \\
(19.4 \%, 63.3 \%)\end{array}$ & Autumn & $\begin{array}{c}-0.73 \%{ }^{* * *} \\
(-1.18 \%,-0.28 \%)\end{array}$ & $\begin{array}{c}-0.60 \%{ }^{* * *} \\
(-1.02 \%,-0.17 \%)\end{array}$ \\
\hline
\end{tabular}

Notes: Annual and seasonal temperature and precipitations marginals at the average U.S. climate measured as a percentage change of land value. 95 percent confidence intervals in parenthesis. Standard errors are corrected for spatial correlation, cutoff point at 3 degrees. $* \mathrm{p}<0.01 ; * * \mathrm{p}<0.05 ; * * * \mathrm{p}<0.001$.

Table 4-The impact of climate change on U.S. agriculture.

\begin{tabular}{|c|c|c|c|c|c|}
\hline & Hsiao & Pooled & & Hsiao & Pooled \\
\hline Billions US\$ & $\begin{array}{c}14.83 \\
(-165.0,208.3)\end{array}$ & $\begin{array}{c}15.21 \\
(-161.2,201.8)\end{array}$ & $\begin{array}{l}\text { Percentage } \\
\text { of land values }\end{array}$ & $\begin{array}{c}1.5 \% \\
(-16.2 \%, 20.5 \%)\end{array}$ & $\begin{array}{c}1.5 \% \\
(-15.8 \%, 19.9 \%)\end{array}$ \\
\hline
\end{tabular}

Notes: Welfare estimates of a uniform $2.7 \mathrm{C}^{\circ}$ warming with an 8 percent increase in precipitation. 95 percentage bootstrap confidence intervals in parenthesis, based on 1,000 repetitions. All dollar figures are in billions of $2000 \mathrm{USD} * \mathrm{p}<0.01 ; * *$ $\mathrm{p}<0.05 ; * * * \mathrm{p}<0.001$.

Table 4 presents the welfare results from the panel regressions. The uniform climate change scenario of a $2.7 \mathrm{C}^{\circ}$ warming with an 8 percent increase in precipitation leads to benefits of $\$ 15$ billion in both the Hsiao and pooled models. This amounts to a 1.5 percent increase in land value.

The confidence intervals for the welfare impacts of climate change in Table 4 have been derived by the bootstrap method using 1,000 repetitions. The welfare estimates have very large error bands around them. This may seem surprising given the tight estimates of the individual 
climate coefficients. However, many of the highly significant seasonal climate variables work in opposite directions so that the net annual effect is small and insignificant.

In order to test whether the panel models do a better job of stabilizing coefficients compared to the repeated cross sectional model, we estimate time varying coefficients with the panel model. In Table 5, we present the Hsiao model with time varying climate coefficients. In Table 6 , we present the pooled model with time varying coefficients. The climate coefficients have the same sign and the same order of magnitude over time. However, the coefficients are not identical. The F-test for whether all the climate coefficients are the same is 8.27; the F-test of the temperature coefficients yields a value of 6.04 and the F-test of the precipitation coefficients yields a value of 3.50. The climate coefficients are not identical. 
Table 5-The time varying Hsiao model

\begin{tabular}{|c|c|c|c|c|c|c|}
\hline Model & 1978 & 1982 & 1987 & 1992 & 1997 & 2002 \\
\hline Temp Winter & $\begin{array}{l}-0.31 \\
(-13)\end{array}$ & $\begin{array}{l}-0.238 \\
(-9.71)\end{array}$ & $\begin{array}{l}-0.213 \\
(-8.83)\end{array}$ & $\begin{array}{l}-0.235 \\
(-9.54)\end{array}$ & $\begin{array}{l}-0.265 \\
(-10.7)\end{array}$ & $\begin{array}{l}-0.288 \\
(-11.5)\end{array}$ \\
\hline Temp Winter sq & $\begin{array}{r}-0.00389 \\
(-4.01)\end{array}$ & $\begin{array}{r}-0.00163 \\
(-1.54)\end{array}$ & $\begin{array}{r}-0.00203 \\
(-1.85)\end{array}$ & $\begin{array}{r}-0.00316 \\
(-2.75)\end{array}$ & $\begin{array}{r}-0.00406 \\
(-3.58)\end{array}$ & $\begin{array}{r}-0.00367 \\
(-3.07)\end{array}$ \\
\hline Temp Spring & $\begin{array}{c}0.496 \\
(7.14)\end{array}$ & $\begin{array}{c}0.423 \\
(6.58)\end{array}$ & $\begin{array}{c}0.325 \\
(4.69)\end{array}$ & $\begin{array}{c}0.326 \\
(4.44)\end{array}$ & $\begin{array}{l}0.339 \\
(5.77)\end{array}$ & $\begin{array}{c}0.314 \\
(4.74)\end{array}$ \\
\hline Temp Spring sq & $\begin{array}{l}-0.0111 \\
(-2.86)\end{array}$ & $\begin{array}{c}-0.00892 \\
(-2.84)\end{array}$ & $\begin{array}{c}-0.00528 \\
(-1.32)\end{array}$ & $\begin{array}{c}-0.00694 \\
(-1.58)\end{array}$ & $\begin{array}{c}-0.00574 \\
(-2.24)\end{array}$ & $\begin{array}{l}-0.00277 \\
(-0.812)\end{array}$ \\
\hline Temp Summer & $\begin{array}{l}-0.819 \\
(-14.9)\end{array}$ & $\begin{array}{l}-0.758 \\
(-13.5)\end{array}$ & $\begin{array}{l}-0.721 \\
(-12.6)\end{array}$ & $\begin{array}{l}-0.713 \\
(-12.6)\end{array}$ & $\begin{array}{l}-0.728 \\
(-13.1)\end{array}$ & $\begin{array}{l}-0.767 \\
(-14)\end{array}$ \\
\hline Temp Summer sq & $\begin{array}{l}0.0099 \\
(7.86)\end{array}$ & $\begin{array}{l}0.0102 \\
(8.22)\end{array}$ & $\begin{array}{c}0.00969 \\
(7.63)\end{array}$ & $\begin{array}{r}0.00957 \\
(7.44)\end{array}$ & $\begin{array}{c}0.00929 \\
(7.69)\end{array}$ & $\begin{array}{c}0.00866 \\
(7.07)\end{array}$ \\
\hline Temp Autumn & $\begin{array}{c}0.263 \\
(2.34)\end{array}$ & $\begin{array}{l}0.212 \\
(1.9)\end{array}$ & $\begin{array}{c}0.238 \\
(1.99)\end{array}$ & $\begin{array}{c}0.223 \\
(1.75)\end{array}$ & $\begin{array}{l}0.254 \\
(2.36)\end{array}$ & $\begin{array}{c}0.375 \\
(3.16)\end{array}$ \\
\hline Temp Autumn sq & $\begin{array}{c}0.00674 \\
(1.3)\end{array}$ & $\begin{array}{c}0.0035 \\
(0.759)\end{array}$ & $\begin{array}{c}0.0015 \\
(0.266)\end{array}$ & $\begin{array}{l}0.00436 \\
(0.704)\end{array}$ & $\begin{array}{l}0.00391 \\
(0.968)\end{array}$ & $\begin{array}{l}0.00063 \\
(0.124)\end{array}$ \\
\hline Prec Winter & $\begin{array}{c}0.00523 \\
(3.59)\end{array}$ & $\begin{array}{r}0.00377 \\
(2.44)\end{array}$ & $\begin{array}{c}0.00693 \\
(4.57)\end{array}$ & $\begin{array}{l}0.0081 \\
(4.97)\end{array}$ & $\begin{array}{l}0.0092 \\
(5.92)\end{array}$ & $\begin{array}{c}0.00973 \\
(5.74)\end{array}$ \\
\hline Prec Winter sq & $\begin{array}{c}-0.00000927 \\
(-1.58)\end{array}$ & $\begin{array}{c}-0.00000909 \\
(-1.41)\end{array}$ & $\begin{array}{c}-0.0000225 \\
(-3.65)\end{array}$ & $\begin{array}{c}-0.0000184 \\
(-2.73)\end{array}$ & $\begin{array}{c}-0.000023 \\
(-3.7)\end{array}$ & $\begin{array}{c}-0.000028 \\
(-4.08)\end{array}$ \\
\hline Prec Spring & $\begin{array}{l}0.0184 \\
(5.39)\end{array}$ & $\begin{array}{l}0.0233 \\
(7.11)\end{array}$ & $\begin{array}{l}0.0162 \\
(4.46)\end{array}$ & $\begin{array}{l}0.0124 \\
(3.22)\end{array}$ & $\begin{array}{l}0.0157 \\
(4.66)\end{array}$ & $\begin{array}{l}0.0215 \\
(5.31)\end{array}$ \\
\hline Prec Spring sq & $\begin{array}{c}-0.000085 \\
(-4.84)\end{array}$ & $\begin{array}{c}-0.0000996 \\
(-6.12)\end{array}$ & $\begin{array}{c}-0.0000853 \\
(-4.78)\end{array}$ & $\begin{array}{c}-0.0000634 \\
(-3.37)\end{array}$ & $\begin{array}{c}-0.0000691 \\
(-4.16)\end{array}$ & $\begin{array}{c}-0.0000902 \\
(-4.66)\end{array}$ \\
\hline Prec Summer & $\begin{array}{r}0.00751 \\
(2.46)\end{array}$ & $\begin{array}{c}0.00319 \\
(1.19)\end{array}$ & $\begin{array}{r}0.00825 \\
(2.62)\end{array}$ & $\begin{array}{l}0.00334 \\
(0.979)\end{array}$ & $\begin{array}{l}0.00156 \\
(0.633)\end{array}$ & $\begin{array}{c}0.00283 \\
(1.05)\end{array}$ \\
\hline Prec Summer sq & $\begin{array}{c}-0.00000905 \\
(-0.52)\end{array}$ & $\begin{array}{c}-0.000000722 \\
(-0.0485)\end{array}$ & $\begin{array}{c}-0.0000242 \\
(-1.33)\end{array}$ & $\begin{array}{c}0.00000802 \\
(0.408)\end{array}$ & $\begin{array}{c}0.0000214 \\
(1.65)\end{array}$ & $\begin{array}{c}0.0000157 \\
(1.08)\end{array}$ \\
\hline Prec Autumn & $\begin{array}{c}-0.0223 \\
(-5.4)\end{array}$ & $\begin{array}{c}-0.0198 \\
(-4.6)\end{array}$ & $\begin{array}{l}-0.0191 \\
(-4.5)\end{array}$ & $\begin{array}{l}-0.0154 \\
(-3.35)\end{array}$ & $\begin{array}{l}-0.0222 \\
(-4.98)\end{array}$ & $\begin{array}{l}-0.0271 \\
(-5.68)\end{array}$ \\
\hline Prec Autumn sq & $\begin{array}{c}0.0000836 \\
(3.67)\end{array}$ & $\begin{array}{c}0.0000765 \\
(3.26)\end{array}$ & $\begin{array}{c}0.0000976 \\
(4.06)\end{array}$ & $\begin{array}{c}0.0000669 \\
(2.51)\end{array}$ & $\begin{array}{c}0.0000919 \\
(3.85)\end{array}$ & $\begin{array}{c}0.000113 \\
(4.28)\end{array}$ \\
\hline & \multicolumn{2}{|c|}{ 1978-2002 } & & & \multicolumn{2}{|c|}{$1978-2002$} \\
\hline Salinity & \multicolumn{2}{|c|}{-0.0913} & \multicolumn{2}{|l|}{ Income } & \multicolumn{2}{|c|}{0.00538} \\
\hline Flooding & \multicolumn{2}{|c|}{$\begin{array}{c}-0.24 \\
(-5.01)\end{array}$} & \multicolumn{2}{|l|}{ Density } & \multicolumn{2}{|c|}{$(6.08)$} \\
\hline Wet index & \multicolumn{2}{|c|}{$\begin{array}{l}0.224 \\
(9.3)\end{array}$} & \multicolumn{2}{|l|}{ Density sq } & \multicolumn{2}{|c|}{$-3.45 E-06$} \\
\hline K-factor & -1 & $(-7.4)$ & \multicolumn{2}{|c|}{ Share of greenhouses } & \multicolumn{2}{|c|}{$\begin{array}{c}0.291 \\
(1.86)\end{array}$} \\
\hline Lenght of slope & \multicolumn{2}{|c|}{0.000119} & \multicolumn{2}{|c|}{ Government transfers } & \multicolumn{2}{|c|}{$(-9.49)$} \\
\hline Sand & \multicolumn{2}{|c|}{0.242} & \multicolumn{2}{|c|}{ House price index } & \multicolumn{2}{|c|}{$(18.2)$} \\
\hline Clay & $\begin{array}{l}-0 \\
(-2\end{array}$ & $\begin{array}{l}106 \\
79)\end{array}$ & 1982 & & $\begin{array}{l}-0 . \\
(-4\end{array}$ & \\
\hline Moisture Level & $\begin{array}{r}1 . \\
(4 .\end{array}$ & $\begin{array}{l}26 \\
49)\end{array}$ & 1987 & & $\begin{array}{l}-0 \\
(-2\end{array}$ & \\
\hline Permeability & $\begin{array}{l}-0.0 \\
(-3\end{array}$ & $\begin{array}{l}519 \\
62)\end{array}$ & 1992 & & $\begin{array}{l}-0 \\
(-4\end{array}$ & \\
\hline Latitude & $\begin{array}{l}-0.0 \\
(-0 .\end{array}$ & $\begin{array}{l}6837 \\
687)\end{array}$ & 1997 & & $\begin{array}{l}-0 \\
(-3\end{array}$ & \\
\hline Elevation & $\begin{array}{r}-0.00 \\
(-5 .\end{array}$ & $\begin{array}{l}0439 \\
33 \text { ) }\end{array}$ & 2002 & & $\begin{array}{l}-0 \\
(-2\end{array}$ & \\
\hline Distance Met Areas & $\begin{array}{l}-0.0 \\
(-17\end{array}$ & $\begin{array}{l}0075 \\
7.3)\end{array}$ & Constant & & & \\
\hline Surface water & $\begin{array}{l}0.0 \\
(22\end{array}$ & $\begin{array}{l}729 \\
.5)\end{array}$ & Adjusted R-s & uared & & \\
\hline
\end{tabular}

Notes: t-statistics in parenthesis. 
Table 6-The Time varying pooled model

\begin{tabular}{|c|c|c|c|c|c|c|}
\hline Model & 1978 & 1982 & 1987 & 1992 & 1997 & 2002 \\
\hline Temp Winter & $\begin{array}{l}-0.338 \\
(-14.9)\end{array}$ & $\begin{array}{c}-0.27 \\
(-11.8)\end{array}$ & $\begin{array}{l}-0.0937 \\
(-4.15)\end{array}$ & $\begin{array}{l}-0.236 \\
(-10.3)\end{array}$ & $\begin{array}{l}-0.254 \\
(-10.9)\end{array}$ & $\begin{array}{l}-0.286 \\
(-12.5)\end{array}$ \\
\hline Temp Winter sq & $\begin{array}{c}-0.00484 \\
(-4.41)\end{array}$ & $\begin{array}{l}-0.00306 \\
(-2.45)\end{array}$ & $\begin{array}{r}-0.00284 \\
(-2.46)\end{array}$ & $\begin{array}{c}-0.00478 \\
(-3.85)\end{array}$ & $\begin{array}{c}-0.00488 \\
(-3.9)\end{array}$ & $\begin{array}{r}-0.00515 \\
(-4.17)\end{array}$ \\
\hline Temp Spring & $\begin{array}{c}0.508 \\
(6.93)\end{array}$ & $\begin{array}{l}0.443 \\
(6.3)\end{array}$ & $\begin{array}{c}0.236 \\
(3.18)\end{array}$ & $\begin{array}{c}0.329 \\
(4.17)\end{array}$ & $\begin{array}{c}0.317 \\
(4.81)\end{array}$ & $\begin{array}{l}0.366 \\
(4.9)\end{array}$ \\
\hline Temp Spring sq & $\begin{array}{l}-0.0124 \\
(-3.19)\end{array}$ & $\begin{array}{l}-0.0109 \\
(-3.36)\end{array}$ & $\begin{array}{c}-0.00658 \\
(-1.53)\end{array}$ & $\begin{array}{c}-0.00954 \\
(-2.07)\end{array}$ & $\begin{array}{c}-0.00701 \\
(-2.55)\end{array}$ & $\begin{array}{c}-0.00762 \\
(-2.06)\end{array}$ \\
\hline Temp Summer & $\begin{array}{l}-0.887 \\
(-7.82)\end{array}$ & $\begin{array}{l}-0.761 \\
(-6.19)\end{array}$ & $\begin{array}{l}-0.283 \\
(-2.49)\end{array}$ & $\begin{array}{l}-0.597 \\
(-4.87)\end{array}$ & $\begin{array}{c}-0.603 \\
(-5.13)\end{array}$ & $\begin{array}{l}-0.647 \\
(-5.6)\end{array}$ \\
\hline Temp Summer sq & $\begin{array}{l}0.0101 \\
(4.33)\end{array}$ & $\begin{array}{r}0.00917 \\
(3.72)\end{array}$ & $\begin{array}{c}0.00253 \\
(1.09)\end{array}$ & $\begin{array}{c}0.00654 \\
(2.56)\end{array}$ & $\begin{array}{l}0.0064 \\
(2.76)\end{array}$ & $\begin{array}{l}0.00551 \\
(2.34)\end{array}$ \\
\hline Temp Autumn & $\begin{array}{l}0.313 \\
(2.7)\end{array}$ & $\begin{array}{c}0.213 \\
(1.83)\end{array}$ & $\begin{array}{c}-0.102 \\
(-0.821)\end{array}$ & $\begin{array}{c}0.135 \\
(1.04)\end{array}$ & $\begin{array}{c}0.188 \\
(1.72)\end{array}$ & $\begin{array}{c}0.229 \\
(1.98)\end{array}$ \\
\hline Temp Autumn sq & $\begin{array}{l}0.00898 \\
(1.65)\end{array}$ & $\begin{array}{c}0.00768 \\
(1.56)\end{array}$ & $\begin{array}{l}0.0104 \\
(1.69)\end{array}$ & $\begin{array}{l}0.0109 \\
(1.71)\end{array}$ & $\begin{array}{l}0.00831 \\
(1.97)\end{array}$ & $\begin{array}{l}0.0094 \\
(1.85)\end{array}$ \\
\hline Prec Winter & $\begin{array}{c}0.00626 \\
(4.5)\end{array}$ & $\begin{array}{c}0.00525 \\
(3.5)\end{array}$ & $\begin{array}{c}0.00751 \\
(5.09)\end{array}$ & $\begin{array}{l}0.00787 \\
(5.02)\end{array}$ & $\begin{array}{c}0.00963 \\
(6.53)\end{array}$ & $\begin{array}{c}0.00576 \\
(3.59)\end{array}$ \\
\hline Prec Winter sq & $\begin{array}{c}-0.0000154 \\
(-2.49)\end{array}$ & $\begin{array}{c}-0.0000133 \\
(-1.94)\end{array}$ & $\begin{array}{c}-0.0000258 \\
(-4.04)\end{array}$ & $\begin{array}{c}-0.0000178 \\
(-2.59)\end{array}$ & $\begin{array}{c}-0.0000244 \\
(-3.87)\end{array}$ & $\begin{array}{c}-0.0000189 \\
(-2.82)\end{array}$ \\
\hline Prec Spring & $\begin{array}{l}0.0161 \\
(4.59)\end{array}$ & $\begin{array}{l}0.0216 \\
(6.4)\end{array}$ & $\begin{array}{c}0.00902 \\
(2.5)\end{array}$ & $\begin{array}{c}0.00987 \\
(2.55)\end{array}$ & $\begin{array}{l}0.0136 \\
(3.95)\end{array}$ & $\begin{array}{l}0.0193 \\
(4.75)\end{array}$ \\
\hline Prec Spring sq & $\begin{array}{c}-0.0000726 \\
(-4.15)\end{array}$ & $\begin{array}{c}-0.0000992 \\
(-6.1)\end{array}$ & $\begin{array}{c}-0.0000636 \\
(-3.45)\end{array}$ & $\begin{array}{c}-0.0000671 \\
(-3.5)\end{array}$ & $\begin{array}{c}-0.0000691 \\
(-4.13)\end{array}$ & $\begin{array}{c}-0.0000898 \\
(-4.59)\end{array}$ \\
\hline Prec Summer & $\begin{array}{c}0.00758 \\
(2.45)\end{array}$ & $\begin{array}{c}0.00342 \\
(1.26)\end{array}$ & $\begin{array}{c}0.00879 \\
(2.62)\end{array}$ & $\begin{array}{c}0.00375 \\
(1.09)\end{array}$ & $\begin{array}{l}0.00154 \\
(0.633)\end{array}$ & $\begin{array}{c}0.0011 \\
(0.418)\end{array}$ \\
\hline Prec Summer sq & $\begin{array}{c}-0.00000583 \\
(-0.329)\end{array}$ & $\begin{array}{c}0.00000472 \\
(0.311)\end{array}$ & $\begin{array}{c}-0.0000386 \\
(-1.96)\end{array}$ & $\begin{array}{c}0.00000696 \\
(0.35)\end{array}$ & $\begin{array}{c}0.0000208 \\
(1.63)\end{array}$ & $\begin{array}{c}0.0000179 \\
(1.27)\end{array}$ \\
\hline Prec Autumn & $\begin{array}{l}-0.0255 \\
(-5.79)\end{array}$ & $\begin{array}{l}-0.0222 \\
(-4.85)\end{array}$ & $\begin{array}{l}-0.0119 \\
(-2.74)\end{array}$ & $\begin{array}{l}-0.0134 \\
(-2.84)\end{array}$ & $\begin{array}{l}-0.021 \\
(-4.57)\end{array}$ & $\begin{array}{l}-0.0209 \\
(-4.49)\end{array}$ \\
\hline Prec Autumn sq & $\begin{array}{c}0.000104 \\
(4.3)\end{array}$ & $\begin{array}{c}0.0000922 \\
(3.7)\end{array}$ & $\begin{array}{c}0.0000893 \\
(3.56)\end{array}$ & $\begin{array}{c}0.0000688 \\
(2.54)\end{array}$ & $\begin{array}{c}0.0000962 \\
(3.95)\end{array}$ & $\begin{array}{c}0.000101 \\
(3.89)\end{array}$ \\
\hline & \multicolumn{2}{|c|}{$1978-2002$} & & & \multicolumn{2}{|c|}{$1978-2002$} \\
\hline Salinity & \multicolumn{2}{|c|}{-0.0971} & \multicolumn{2}{|l|}{ Income } & \multicolumn{2}{|c|}{$(5.67)$} \\
\hline Flooding & \multicolumn{2}{|c|}{$\begin{array}{c}-0.249 \\
(-5.92)\end{array}$} & Density & & \multicolumn{2}{|c|}{$(10.9)$} \\
\hline Wet index & \multicolumn{2}{|c|}{$\begin{array}{c}0.114 \\
(4.74)\end{array}$} & Density sq & & \multicolumn{2}{|c|}{-0.00000125} \\
\hline K-factor & \multicolumn{2}{|c|}{-0.966} & Share of gre & houses & \multicolumn{2}{|c|}{ (3.35) } \\
\hline Lenght of slope & 0.000086 & (2.1) & Government & ansfers & \multicolumn{2}{|c|}{$\begin{array}{c}0.00454 \\
(22.3)\end{array}$} \\
\hline Sand & \multicolumn{2}{|c|}{$(1.08)$} & House price & dex & \multicolumn{2}{|c|}{0.00558} \\
\hline Clay & \multicolumn{2}{|c|}{-0.119} & 1982 & & \multicolumn{2}{|c|}{-0.351} \\
\hline Moisture Level & $\begin{array}{l}0.4 \\
(1 .\end{array}$ & & 1987 & & & \\
\hline Permeability & $\begin{array}{l}-0.0 \\
(-3\end{array}$ & & 1992 & & $\begin{array}{l}-0 \\
(-0\end{array}$ & \\
\hline Latitude & $\begin{array}{l}-0.0 \\
(-0 .\end{array}$ & $\begin{array}{l}776 \\
47)\end{array}$ & 1997 & & $\begin{array}{l}-1 \\
(-0\end{array}$ & 22 \\
\hline Elevation & $\begin{array}{r}-0.00 \\
(-5 .\end{array}$ & $\begin{array}{l}459 \\
8 \text { ) }\end{array}$ & 2002 & & $\begin{array}{l}-0 \\
(-0\end{array}$ & $\begin{array}{l}68 \\
41)\end{array}$ \\
\hline Distance Met Areas & $\begin{array}{r}-0.00 \\
(-1 \delta\end{array}$ & $\begin{array}{l}807 \\
5)\end{array}$ & Constant & & & \\
\hline Surface water & $\begin{array}{l}0.0 \\
(21\end{array}$ & & Adjusted R-s & uared & & \\
\hline
\end{tabular}

Notes: t-statistics in parenthesis. 
Table 7-Welfare estimates of climate change impacts

\begin{tabular}{cccc}
\hline \hline Model & Hsiao time varying & Pooled time varying & $\begin{array}{c}\text { Enhanced repeated } \\
\text { cross section }\end{array}$ \\
\hline 1978 & 31.87 & 9.47 & 127.32 \\
1982 & $(3.1 \%)$ & $(0.9 \%)$ & $(12.5 \%)$ \\
1987 & 9.15 & 3.91 & 98.31 \\
1992 & $(0.9 \%)$ & $(0.4 \%)$ & $(9.6 \%)$ \\
1997 & 6.47 & 18.44 & -52.31 \\
& $(0.6 \%)$ & $(1.8 \%)$ & $-5.1 \%)$ \\
2002 & 18.32 & 5.51 & $(5.3 \%)$ \\
& $(1.8 \%)$ & $(1.8 \%)$ & 15.17 \\
& 28.33 & 19.49 & $(1.5 \%)$ \\
\hline
\end{tabular}

Notes: All dollar figures are in billions of 2000 USD. Percentage impacts are in parenthesis. Welfare impacts correspond to a uniform increase of temperature of $2.7^{\circ} \mathrm{C}$ and of 8 percent of precipitation.

In Table 7, we present the welfare results with the time varying climate coefficients. We also show the results of the repeated cross section for comparison. The Hsiao panel regression results are more robust than the repeated cross section. The pooled results are especially stable across time. Despite the fact that the Hsiao model does a better job of controlling for missing cross sectional variables, the pooled model givens the best results.

\section{v. Conclusion}

This paper examines the possibility of using panel data to estimate cross sectional valuation models such as the Ricardian model. Repeated cross sectional analyses such as presented in DG are not robust. We argue that this is because they are mis-specified. The coefficients of time varying and time invariant variables are allowed to vary over time in the repeated cross sections. We argue the correctly specified model holds these coefficients constant. 
We explore two panel data models that are properly specified. The result is a highly significant model that predicts climate coefficients with a high degree of accuracy. In order to test whether the panel data models have done a good job of stabilizing climate estimates across years, we estimate year-specific climate effects. Whereas the repeated cross sectional models produce widely varying effects, the two panel models predict much more stable results. The pooled model produces the best results.

DG use their analysis of the Ricardian model to advocate the use of intertemporal weather analysis. However, it is important to recognize that the Ricardian and intertemporal models are not measuring the same thing. The relationship between annual farm profits and weather is an unbiased measure of the impact of abrupt climate change or weather. However, analyzing weather effects does not capture adaptation and so it is a biased (too pessimistic) measure of the long term effects of climate change. The Ricardian approach, in turn, provides a biased (too optimistic) estimate of the impact of abrupt climate change or weather. But the Ricardian method captures adaptation and so it is an unbiased estimate of the impact of long term climate change.

The results of the analysis of weather and climate are quite different. Whereas every farmer in the world is concerned about weather, the effect of climate will vary a great deal depending on where a farm is located. Farms in relatively cool locations will benefit but farms in warmer locations are likely to be hurt. Even within the United States, farms in the northern US may see large benefits whereas farms along the southern edges of the US will be harmed. The analysis also suggests that farms in the Western US will be hurt. The losses can be even larger in tropical countries in Africa (Kurukulasuriya et al. 2005) or Latin America (Seo and Mendelsohn 2008c). 
This study develops a panel data method for estimating Ricardian models. The results reveal that the climate coefficients are far more accurate than coefficients from repeated cross sections. The results suggest that many cross sectional valuation methods may benefit from panel data provided they estimate their panel data models correctly. 


\section{Data Appendix}

We have constructed a balanced panel with observations for 2,914 counties in the contiguous 48 States over the years 1978, 1982, 1987, 1992, 1997 and 2002. Units of measurement are in the metric system; economic variables have all been converted to constant 2000 USD using the GDP deflator. If not otherwise stated, variables measure data of interest in years 1978, 1982, 1987, 1992, 1997 and 2002.

\section{Time Varying, County Specific Socio-Economic Variables}

Farmland Value - Estimated Value of Land and Buildings, average per hectare of farmland. Data source is the Agricultural Census.

Farmland - Land in farms as in the Census of Agriculture from 1978 to 2002, hectares. The Census of Agriculture defines "Land in farms"' as agricultural land used for crops, pasture, or grazing. It also includes woodland and wasteland not actually under cultivation or used for pasture or grazing, provided it was part of the farm operator's total operation. Large acreages of woodland or wasteland held for nonagricultural purposes were deleted from individual reports. Land in farms includes acres in the Conservation Reserve and Wetlands Reserve Programs. Land in farms is an operating unit concept and includes land owned and operated as well as land rented from others.

Income - Per capita personal income, (\$); Bureau of Economic Analysis, Regional Economic Accounts, table CA1-3.

Density - Population per squared kilometre, Bureau of Economic Analysis, Regional Economic Accounts, table CA1-3. 
Greenhouses - Share of total crop sales from nursery, greenhouse and floriculture. Source: Census of Agriculture.

Subsidies - Government payments per hectare of land (\$). Source: Census of Agriculture.

Real Estate - Median value for all owner-occupied housing units (1,000 \$). Census of Population and Housing, 1980, 1990 and 2000. Values for panel years have been computed extrapolating linear trends from the three census years available.

\section{Time InVARIant, County SPecific, Climate Variables}

Climate Variables - We have estimated quadratic climate surfaces for the contiguous 48 States following the approach described in MNS (1994). The data source for climate normals is the National Climatic Data Center CLIM81 series, which contains monthly station normals (19712000) for 7,467 weather stations in the contiguous 48 States, of which 2,135 provide only precipitation data. For each county we select weather stations contained in a 500 miles radius circle centered at the county's centroid and we estimate local monthly climate by applying Weighted Least Squares to a quadratic combination of stations' longitude, latitude, elevation, and distance from coastline; as weights we use the squared distance between the county's centroid and the weather station. Seasonal climate is obtained as the arithmetic average of climate variables in Winter (December, January, February), Spring (March, April, May), Summer (June, July, August) and Autumn (September, October, November). Temperatures are measured in degrees Celsius and precipitations are measured in millimeters. 


\section{TIME INVARIANT, COUNTY SPECIFIC SOIL CHARACTERISTICS}

Salinity - Percentage of agricultural land that has salinity-sodium problems.

Flooding - Percentage of agricultural land occasionally or frequently prone to flooding.

Wet Factor - Percentage of agricultural land that has very low drainage (Poor and Very Poor).

Kfactor - Average soil erodibility factor. It is the average soil loss in tons/acre; is a measure of the susceptibility of soil particles to detachment and transport by rainfall and runoff.

Slope Length - Average slope length factor, feet.

Sand - Percentage of agricultural land classified as sand or coarse-textured soils.

Clay - Percentage of agricultural land that is classified as clay.

Moisture Level - Minimum value for the range of available water capacity for the soil layer or horizon, expressed as inches/inch.

Permeability - The minimum value for the range in permeability rate for the soil layer or horizon, expressed as inches/hour.

\section{Time InVARIant, COUnTy Specific GeograPhic Variables}

Latitude - Latitude of county's centroid in decimal degrees.

Elevation - Elevation of county's centroid in meters.

Distance from Metropolitan Areas - Distance in kilometers between county's centroid and metropolitan areas with more than 200,000 inhabitants in 2000 . 
Surface Water Withdrawal - Thousands of liters per hectare, per day, of surface fresh water for irrigation purposes. The "Estimated Use of Water in the United States", published every five years by the United States Geological Survey (USGS), supplies data on water use at county level only starting from 1985 . We divided the amount of water used at county level for years 1985 , 1990, 1995, 2000 by the amount of farmland in that county in Census years 1987, 1992, 1997 and 2002, respectively, and we computed the time average of surface water use per hectare of land. We used this variable as a proxy for surface water availability, at county level, for all time observations of our panel. 


\section{References}

Conley, Timothy, "GMM Estimation with Cross Sectional Dependence." Journal of Econometrics 92:1 (1999), 1-45.

Deschenes, Oliver, and Michael Greenstone, "The Economic Impacts of Climate Change: Evidence from Agricultural Output and Random Fluctuations in Weather." American Economic Review 97:1 (2007), 354-385.

Fisher, Anthony, Michael Hanemann, Michael Roberts and Wolfram Schlenker, "Potential Impacts of Climate Change on Crop Yields and Land Values in U.S. Agriculture: Negative, Significant, and Robust.” American Economic Review (2010), forthcoming.

Hsiao, Cheng, Analysis of Panel Data (Cambridge: Cambridge University Press, 2nd Edition, 2008).

Kurukulasuriya, Pradeep and Robert Mendelsohn, “Crop Switching as an Adaptation Strategy to Climate Change." African Journal Agriculture and Resource Economics 2:2 (2008), 105-126.

Kurukulasuriya, Pradeep, Robert Mendelsohn, Rashid Hassan, James Benhin, Temesgen Deressa, Mbaye Diop, Helmy Mohamed Eid, K. Yerfi Fosu, Glwadys Gbetibouo, Suman Jain, Ali Mahamadou, Rene Mano, Jane Kabubo-Mariara, Samia El-Marsafawy, Ernest Molua, Samiha Ouda, Mathieu Ouedraogo, Isidor Sène, David Maddison, Niggol Seo and Ariel Dinar,

“Will African Agriculture Survive Climate Change?” World Bank Economic Review 20:3 (2006), $367-388$. 
Mendelsohn, R., A. Dinar and A. Sanghi, "The Effect of Development on the Climate Sensitivity of Agriculture," Environment and Development Economics 6 (2001); 85-101.

Mendelsohn, Robert and Ariel Dinar, "Climate, Water, and Agriculture." Land Economics 79:3 (2003), 328-341.

Mendelsohn, Robert and Ariel Dinar, Climate Change and Agriculture: An Economic Analysis of Global Impacts, Adaptation, and Distributional Effects (Cheltenham, UK: Edward Elgar Publishing, 2009).

Mendelsohn, Robert and Sheila Olmstead, "The Economic Valuation of Environmental Amenities and Disamenities: Methods and Applications." Annual Review of Environment and Resources 34 (2009), 325-347.

Mendelsohn, Robert, William Nordhaus and Daigee Shaw, "Measuring the Impact of Global Warming on Agriculture," American Economic Review 84:4 (1994), 753-771.

Mendelsohn, Robert, William Nordhaus and Daigee Shaw, "Climate Impacts on Aggregate Farm Values: Accounting for Adaptation," Agriculture and Forest Meteorology 80:1 (1996), 55-67.

Mrozek, Janusz R. and Laura O. Taylor, "What Determines the Value of Life? A MetaAnalysis," Journal of Policy Analysis Management 21:2 (2002), 253-270.

Palmquist, Raymond and Kerry V. Smith, "The Use of Hedonic Property Value Techniques for Policy and Litigation," in T. Tietenberg and H. Folmer (Eds.), The International Yearbook of Environmental and Resource Economics 2002/2003 Vol. 3, 115-64 (Cheltenham, UK: Edward Elgar 2003). 
Parsons, George R. and Yangru Wu, “The Opportunity Cost of Coastal Land-Use Controls: An Empirical Analysis," Land Economics 67:3 (1991), 308-316.

Sanghi, A. and R. Mendelsohn, “ The Impacts Of Global Warming On Farmers In Brazil And India," Global Environmental Change 18 (2008), 655-665.

Schlenker, Wolfram, Michael Hanemann and Anthony Fischer, "Will US Agriculture Really Benefit from Global Warming? Accounting for Irrigation in the Hedonic Approach," American Economic Review 95:1 (2005), 395-406.

Seo, Niggol and Robert Mendelsohn, “An Analysis of Crop Choice: Adapting to Climate Change in Latin American Farms,” Ecological Economics 67:1 (2008a), 109-116.

Seo, Niggol and Robert Mendelsohn, "Measuring Impacts and Adaptation to Climate Change: A Structural Ricardian Model of African Livestock Management," Agricultural Economics 38:2 (2008b), 150-165.

Seo, Niggol and Robert Mendelsohn, "A Ricardian Analysis of the Impact of Climate Change on South American Farms," Chilean Journal Of Agricultural Research 68:1 (2008c), 69-79.

Viscusi, W. Kip and Joseph Aldy, "The Value of a Statistical Life: A Critical Review of Market Estimates Throughout the World.” Journal of Risk Uncertainty 27:1 (2003), 5-76. 


\section{Appendix}

Table A1- Enhanced repeated cross section model

\begin{tabular}{|c|c|c|c|c|c|c|c|c|c|c|c|c|c|}
\hline Model & 1978 & 1982 & 1987 & 1992 & 1997 & 2002 & & 1978 & 1982 & 1987 & 1992 & 1997 & 2002 \\
\hline Temp Winter & $\begin{array}{c}-3.22 \mathrm{E}-01 \\
(-7.05)\end{array}$ & $\begin{array}{c}-2.74 \mathrm{E}-01 \\
(-6.26)\end{array}$ & $\begin{array}{c}-1.54 \mathrm{E}-01 \\
(-3.61)\end{array}$ & $\begin{array}{c}-2.44 \mathrm{E}-01 \\
(-5.27)\end{array}$ & $\begin{array}{c}-2.27 \mathrm{E}-01 \\
(-5.52)\end{array}$ & $\begin{array}{c}-2.68 \mathrm{E}-01 \\
(-6.57)\end{array}$ & Salinity & $\begin{array}{c}-6.73 \mathrm{E}-02 \\
(-0.74)\end{array}$ & $\begin{array}{c}-1.23 \mathrm{E}-01 \\
(-1.32)\end{array}$ & $\begin{array}{c}-1.23 \mathrm{E}-01 \\
(-1.29)\end{array}$ & $\begin{array}{c}-1.03 \mathrm{E}-01 \\
(-1.02)\end{array}$ & $\begin{array}{c}-9.49 \mathrm{E}-02 \\
(-0.93)\end{array}$ & $\begin{array}{c}-1.89 \mathrm{E}-02 \\
(-0.18)\end{array}$ \\
\hline Temp Winter sq & $\begin{array}{c}-6.61 \mathrm{E}-03 \\
(-3.73)\end{array}$ & $\begin{array}{c}-6.20 \mathrm{E}-03 \\
(-3.24)\end{array}$ & $\begin{array}{c}-3.45 \mathrm{E}-03 \\
(-1.82)\end{array}$ & $\begin{array}{c}-6.18 \mathrm{E}-03 \\
(-3.1)\end{array}$ & $\begin{array}{c}-4.99 \mathrm{E}-03 \\
(-2.65)\end{array}$ & $\begin{array}{c}-4.53 \mathrm{E}-03 \\
(-2.39)\end{array}$ & Flooding & $\begin{array}{c}-3.36 \mathrm{E}-01 \\
(-3.25)\end{array}$ & $\begin{array}{c}-2.72 \mathrm{E}-01 \\
(-2.51)\end{array}$ & $\begin{array}{c}-2.13 \mathrm{E}-01 \\
(-2.59)\end{array}$ & $\begin{array}{c}-2.32 \mathrm{E}-01 \\
(-2.16)\end{array}$ & $\begin{array}{c}-2.06 \mathrm{E}-01 \\
(-1.96)\end{array}$ & $\begin{array}{c}-2.14 \mathrm{E}-01 \\
(-2.64)\end{array}$ \\
\hline Temp Spring & $\begin{array}{c}3.79 \mathrm{E}-01 \\
(3.76)\end{array}$ & $\begin{array}{c}4.30 \mathrm{E}-01 \\
(4.44)\end{array}$ & $\begin{array}{c}3.02 \mathrm{E}-01 \\
(3.52)\end{array}$ & $\begin{array}{c}4.54 \mathrm{E}-01 \\
(4.38)\end{array}$ & $\begin{array}{c}3.65 \mathrm{E}-01 \\
(3.35)\end{array}$ & $\begin{array}{c}3.01 \mathrm{E}-01 \\
(2.42)\end{array}$ & Wet index & $\begin{array}{c}4.08 \mathrm{E}-01 \\
(4.05)\end{array}$ & $\begin{array}{c}2.18 \mathrm{E}-01 \\
(2.3)\end{array}$ & $\begin{array}{c}-7.97 \mathrm{E}-02 \\
(-1)\end{array}$ & $\begin{array}{c}-8.80 \mathrm{E}-03 \\
(-0.09)\end{array}$ & $\begin{array}{c}-2.44 \mathrm{E}-02 \\
(-0.24)\end{array}$ & $\begin{array}{c}-1.76 \mathrm{E}-02 \\
(-0.18)\end{array}$ \\
\hline Temp Spring sq & $\begin{array}{c}-9.92 \mathrm{E}-03 \\
(-2.16)\end{array}$ & $\begin{array}{c}-1.22 \mathrm{E}-02 \\
(-2.9)\end{array}$ & $\begin{array}{c}-8.84 \mathrm{E}-03 \\
(-2.25)\end{array}$ & $\begin{array}{c}-1.41 \mathrm{E}-02 \\
(-3.05)\end{array}$ & $\begin{array}{c}-9.23 \mathrm{E}-03 \\
(-2.03)\end{array}$ & $\begin{array}{c}-5.27 \mathrm{E}-03 \\
(-1.02)\end{array}$ & $\mathrm{K}$-factor & $\begin{array}{c}-4.66 \mathrm{E}-01 \\
(-1.14)\end{array}$ & $\begin{array}{c}-8.11 \mathrm{E}-01 \\
(-2.07)\end{array}$ & $\begin{array}{c}-5.98 \mathrm{E}-01 \\
(-1.85)\end{array}$ & $\begin{array}{c}-1.01 \mathrm{E}+00 \\
(-2.57)\end{array}$ & $\begin{array}{c}-1.32 \mathrm{E}+00 \\
(-3.72)\end{array}$ & $\begin{array}{c}-1.30 E+00 \\
(-3.44)\end{array}$ \\
\hline Temp Summer & $\begin{array}{c}-7.18 \mathrm{E}-01 \\
(-4.63)\end{array}$ & $\begin{array}{c}-5.81 \mathrm{E}-01 \\
(-3.46)\end{array}$ & $\begin{array}{c}-4.25 \mathrm{E}-01 \\
(-2.16)\end{array}$ & $\begin{array}{c}-5.68 \mathrm{E}-01 \\
(-2.81)\end{array}$ & $\begin{array}{c}-4.81 \mathrm{E}-01 \\
(-2.78)\end{array}$ & $\begin{array}{c}-6.26 \mathrm{E}-01 \\
(-3.82)\end{array}$ & Lenght of slope & $\begin{array}{c}3.46 \mathrm{E}-05 \\
(0.34)\end{array}$ & $\begin{array}{c}3.99 \mathrm{E}-05 \\
(0.41)\end{array}$ & $\begin{array}{c}3.43 \mathrm{E}-05 \\
(0.33)\end{array}$ & $\begin{array}{c}4.21 \mathrm{E}-05 \\
(0.38)\end{array}$ & $\begin{array}{c}1.76 \mathrm{E}-04 \\
(1.86)\end{array}$ & $\begin{array}{c}1.61 \mathrm{E}-04 \\
(1.43)\end{array}$ \\
\hline Temp Summer sq & $\begin{array}{c}6.60 \mathrm{E}-03 \\
(2.05)\end{array}$ & $\begin{array}{c}5.29 \mathrm{E}-03 \\
(1.54)\end{array}$ & $\begin{array}{c}4.41 \mathrm{E}-03 \\
(1.09)\end{array}$ & $\begin{array}{c}5.86 \mathrm{E}-03 \\
(1.38)\end{array}$ & $\begin{array}{c}4.04 \mathrm{E}-03 \\
(1.16)\end{array}$ & $\begin{array}{c}5.13 \mathrm{E}-03 \\
(1.48)\end{array}$ & Sand & $\begin{array}{c}2.75 \mathrm{E}-01 \\
(1.72)\end{array}$ & $\begin{array}{c}3.10 \mathrm{E}-02 \\
(0.23)\end{array}$ & $\begin{array}{c}-5.46 \mathrm{E}-02 \\
(-0.39)\end{array}$ & $\begin{array}{c}-2.25 \mathrm{E}-01 \\
(-1.39)\end{array}$ & $\begin{array}{c}-1.19 \mathrm{E}-01 \\
(-0.71)\end{array}$ & $\begin{array}{c}7.70 \mathrm{E}-03 \\
(0.04)\end{array}$ \\
\hline Temp Autumn & $\begin{array}{c}3.12 \mathrm{E}-01 \\
(1.48)\end{array}$ & $\begin{array}{c}7.15 \mathrm{E}-02 \\
(0.34)\end{array}$ & $\begin{array}{c}-5.78 \mathrm{E}-02 \\
(-0.28)\end{array}$ & $\begin{array}{c}-9.00 \mathrm{E}-02 \\
(-0.38)\end{array}$ & $\begin{array}{c}3.75 \mathrm{E}-02 \\
(0.16)\end{array}$ & $\begin{array}{c}3.06 \mathrm{E}-01 \\
(1.32)\end{array}$ & Clay & $\begin{array}{c}-1.73 \mathrm{E}-01 \\
(-1.75)\end{array}$ & $\begin{array}{c}-1.13 \mathrm{E}-01 \\
(-1.42)\end{array}$ & $\begin{array}{c}5.16 \mathrm{E}-02 \\
(0.62)\end{array}$ & $\begin{array}{c}-8.44 \mathrm{E}-02 \\
(-0.93)\end{array}$ & $\begin{array}{c}-2.73 E-01 \\
(-2.55)\end{array}$ & $\begin{array}{c}-2.73 \mathrm{E}-01 \\
(-2.02)\end{array}$ \\
\hline Temp Autumn sq & $\begin{array}{c}1.12 \mathrm{E}-02 \\
(1.64)\end{array}$ & $\begin{array}{c}1.56 \mathrm{E}-02 \\
(2.38)\end{array}$ & $\begin{array}{c}1.16 \mathrm{E}-02 \\
(1.7)\end{array}$ & $\begin{array}{c}1.83 \mathrm{E}-02 \\
(2.49)\end{array}$ & $\begin{array}{c}1.27 \mathrm{E}-02 \\
(1.82)\end{array}$ & $\begin{array}{c}6.64 \mathrm{E}-03 \\
(0.89)\end{array}$ & Moisture Level & $\begin{array}{c}7.09 \mathrm{E}-01 \\
(0.85)\end{array}$ & $\begin{array}{c}3.61 \mathrm{E}-01 \\
(0.41)\end{array}$ & $\begin{array}{c}-7.91 \mathrm{E}-01 \\
(-1.1)\end{array}$ & $\begin{array}{c}-1.42 \mathrm{E}-01 \\
(-0.16)\end{array}$ & $\begin{array}{c}-9.53 \mathrm{E}-01 \\
(-1.11)\end{array}$ & $\begin{array}{c}-6.52 \mathrm{E}-02 \\
(-0.08)\end{array}$ \\
\hline Prec Winter & $\begin{array}{c}7.99 \mathrm{E}-03 \\
(3.06)\end{array}$ & $\begin{array}{c}7.11 \mathrm{E}-03 \\
(2.72)\end{array}$ & $\begin{array}{c}7.38 \mathrm{E}-03 \\
(2.83)\end{array}$ & $\begin{array}{c}8.01 \mathrm{E}-03 \\
(2.79)\end{array}$ & $\begin{array}{c}8.81 \mathrm{E}-03 \\
(3.18)\end{array}$ & $\begin{array}{c}4.12 \mathrm{E}-03 \\
(1.42)\end{array}$ & Permeability & $\begin{array}{c}-8.48 \mathrm{E}-02 \\
(-2.26)\end{array}$ & $\begin{array}{c}-3.22 \mathrm{E}-02 \\
(-1.51)\end{array}$ & $\begin{array}{c}-3.25 \mathrm{E}-02 \\
(-1.38)\end{array}$ & $\begin{array}{c}-6.13 \mathrm{E}-03 \\
(-0.27)\end{array}$ & $\begin{array}{c}-5.45 \mathrm{E}-02 \\
(-1.73)\end{array}$ & $\begin{array}{c}-6.43 \mathrm{E}-02 \\
(-1.71)\end{array}$ \\
\hline Prec Winter sq & $\begin{array}{c}-2.07 \mathrm{E}-05 \\
(-2.24)\end{array}$ & $\begin{array}{c}-1.54 \mathrm{E}-05 \\
(-1.64)\end{array}$ & $\begin{array}{c}-2.16 \mathrm{E}-05 \\
(-2.25)\end{array}$ & $\begin{array}{c}-1.48 \mathrm{E}-05 \\
(-1.46)\end{array}$ & $\begin{array}{c}-2.37 \mathrm{E}-05 \\
(-2.38)\end{array}$ & $\begin{array}{c}-1.83 \mathrm{E}-05 \\
(-1.76)\end{array}$ & Distance metro areas & $\begin{array}{c}-6.78 \mathrm{E}-04 \\
(-3.61)\end{array}$ & $\begin{array}{c}-8.31 \mathrm{E}-04 \\
(-4.84)\end{array}$ & $\begin{array}{c}-7.09 \mathrm{E}-04 \\
(-4.46)\end{array}$ & $\begin{array}{c}-9.66 \mathrm{E}-04 \\
(-5.39)\end{array}$ & $\begin{array}{c}-8.96 \mathrm{E}-04 \\
(-5.05)\end{array}$ & $\begin{array}{c}-8.28 \mathrm{E}-04 \\
(-4.37)\end{array}$ \\
\hline Prec Spring & $\begin{array}{c}9.63 \mathrm{E}-03 \\
(2.05)\end{array}$ & $\begin{array}{c}1.74 \mathrm{E}-02 \\
(3.92)\end{array}$ & $\begin{array}{c}1.06 \mathrm{E}-02 \\
(2.32)\end{array}$ & $\begin{array}{c}8.72 \mathrm{E}-03 \\
(1.68)\end{array}$ & $\begin{array}{c}1.27 \mathrm{E}-02 \\
(2.57)\end{array}$ & $\begin{array}{c}1.87 \mathrm{E}-02 \\
(3.29)\end{array}$ & Surface water & $\begin{array}{c}6.40 \mathrm{E}-02 \\
(8.15)\end{array}$ & $\begin{array}{c}7.02 \mathrm{E}-02 \\
(8.72)\end{array}$ & $\begin{array}{c}6.98 \mathrm{E}-02 \\
(7.81)\end{array}$ & $\begin{array}{c}7.24 \mathrm{E}-02 \\
(8.04)\end{array}$ & $\begin{array}{c}7.63 \mathrm{E}-02 \\
(8.35)\end{array}$ & $\begin{array}{c}7.56 \mathrm{E}-02 \\
(7.83)\end{array}$ \\
\hline Prec Spring sq & $\begin{array}{c}-4.49 \mathrm{E}-05 \\
(-1.75)\end{array}$ & $\begin{array}{c}-1.01 \mathrm{E}-04 \\
(-4.16)\end{array}$ & $\begin{array}{c}-7.07 \mathrm{E}-05 \\
(-2.79)\end{array}$ & $\begin{array}{c}-7.23 \mathrm{E}-05 \\
(-2.49)\end{array}$ & $\begin{array}{c}-6.68 \mathrm{E}-05 \\
(-2.42)\end{array}$ & $\begin{array}{c}-7.58 \mathrm{E}-05 \\
(-2.56)\end{array}$ & Income & $\begin{array}{c}8.67 \mathrm{E}-03 \\
(1.13)\end{array}$ & $\begin{array}{c}-3.59 \mathrm{E}-03 \\
(-0.57)\end{array}$ & $\begin{array}{c}1.85 \mathrm{E}-02 \\
(3.61)\end{array}$ & $\begin{array}{c}1.63 \mathrm{E}-02 \\
(2.96)\end{array}$ & $\begin{array}{c}1.90 \mathrm{E}-02 \\
(3.37)\end{array}$ & $\begin{array}{c}3.61 \mathrm{E}-03 \\
(0.63)\end{array}$ \\
\hline Prec Summer & $\begin{array}{c}7.30 \mathrm{E}-03 \\
(1.58)\end{array}$ & $\begin{array}{c}4.83 \mathrm{E}-03 \\
(1.22)\end{array}$ & $\begin{array}{c}5.03 \mathrm{E}-03 \\
(1.17)\end{array}$ & $\begin{array}{c}2.73 \mathrm{E}-03 \\
(0.59)\end{array}$ & $\begin{array}{c}1.71 \mathrm{E}-03 \\
(0.43)\end{array}$ & $\begin{array}{c}2.77 \mathrm{E}-03 \\
(0.67)\end{array}$ & Density & $\begin{array}{c}2.11 \mathrm{E}-03 \\
(4.5)\end{array}$ & $\begin{array}{c}1.90 \mathrm{E}-03 \\
(3.92)\end{array}$ & $\begin{array}{c}2.03 \mathrm{E}-03 \\
(4.34)\end{array}$ & $\begin{array}{c}2.00 \mathrm{E}-03 \\
(3.57)\end{array}$ & $\begin{array}{c}1.46 \mathrm{E}-03 \\
(2.78)\end{array}$ & $\begin{array}{c}1.90 \mathrm{E}-03 \\
(3.42)\end{array}$ \\
\hline Prec Summer sq & $\begin{array}{c}-7.60 \mathrm{E}-06 \\
(-0.3)\end{array}$ & $\begin{array}{c}2.82 \mathrm{E}-06 \\
(0.13)\end{array}$ & $\begin{array}{c}-1.23 \mathrm{E}-05 \\
(-0.51)\end{array}$ & $\begin{array}{c}1.08 \mathrm{E}-05 \\
(0.42)\end{array}$ & $\begin{array}{c}1.43 \mathrm{E}-05 \\
(0.67)\end{array}$ & $\begin{array}{c}1.12 \mathrm{E}-05 \\
(0.52)\end{array}$ & Density sq & $\begin{array}{r}-1.1 \mathrm{E}-06 \\
(-2.58)\end{array}$ & $\begin{array}{c}-1.21 \mathrm{E}-06 \\
(-2.28)\end{array}$ & $\begin{array}{c}-1.3 \mathrm{E}-06 \\
(-2.48)\end{array}$ & $\begin{array}{c}-1.35 \mathrm{E}-06 \\
(-2.22)\end{array}$ & $\begin{array}{c}-1.28 \mathrm{E}-06 \\
(-2.4)\end{array}$ & $\begin{array}{c}-1.4 \mathrm{E}-06 \\
(-2.7)\end{array}$ \\
\hline Prec Autumn & $\begin{array}{c}-2.42 \mathrm{E}-02 \\
(-4.02)\end{array}$ & $\begin{array}{c}-2.13 \mathrm{E}-02 \\
(-3.59)\end{array}$ & $\begin{array}{c}-1.46 \mathrm{E}-02 \\
(-2.6)\end{array}$ & $\begin{array}{c}-1.30 \mathrm{E}-02 \\
(-2.1)\end{array}$ & $\begin{array}{c}-1.74 \mathrm{E}-02 \\
(-2.9)\end{array}$ & $\begin{array}{c}-1.93 \mathrm{E}-02 \\
(-2.98)\end{array}$ & Share of greenhouse: & $\begin{array}{c}2.19 \mathrm{E}-01 \\
(0.95)\end{array}$ & $\begin{array}{c}4.21 \mathrm{E}-01 \\
(1.98)\end{array}$ & $\begin{array}{c}4.47 \mathrm{E}-01 \\
(2.94)\end{array}$ & $\begin{array}{c}2.98 \mathrm{E}-01 \\
(1.39)\end{array}$ & $\begin{array}{c}5.46 \mathrm{E}-01 \\
(3.24)\end{array}$ & $\begin{array}{c}2.29 \mathrm{E}-01 \\
(1.76)\end{array}$ \\
\hline Prec Autumn sq & $\begin{array}{c}1.02 \mathrm{E}-04 \\
(3.18)\end{array}$ & $\begin{array}{c}9.49 \mathrm{E}-05 \\
(3.04)\end{array}$ & $\begin{array}{c}9.21 \mathrm{E}-05 \\
(2.93)\end{array}$ & $\begin{array}{c}6.94 \mathrm{E}-05 \\
(2.03)\end{array}$ & $\begin{array}{c}8.95 \mathrm{E}-05 \\
(2.69)\end{array}$ & $\begin{array}{c}9.53 \mathrm{E}-05 \\
(2.7)\end{array}$ & Government transfers & $\begin{array}{c}1.55 \mathrm{E}-02 \\
(6.46)\end{array}$ & $\begin{array}{c}1.94 \mathrm{E}-02 \\
(7.22)\end{array}$ & $\begin{array}{c}2.92 \mathrm{E}-03 \\
(6.74)\end{array}$ & $\begin{array}{c}6.05 \mathrm{E}-03 \\
(4.17)\end{array}$ & $\begin{array}{c}1.01 \mathrm{E}-02 \\
(5.84)\end{array}$ & $\begin{array}{c}3.68 \mathrm{E}-03 \\
(3.73)\end{array}$ \\
\hline Latitude & $\begin{array}{c}-7.14 \mathrm{E}-03 \\
(-0.2)\end{array}$ & $\begin{array}{c}2.94 \mathrm{E}-03 \\
(0.09)\end{array}$ & $\begin{array}{c}-3.34 \mathrm{E}-02 \\
(-1.09)\end{array}$ & $\begin{array}{c}-3.05 \mathrm{E}-02 \\
(-0.83)\end{array}$ & $\begin{array}{c}-3.00 \mathrm{E}-03 \\
(-0.08)\end{array}$ & $\begin{array}{c}1.75 \mathrm{E}-02 \\
(0.47)\end{array}$ & House price index & $\begin{array}{c}6.08 \mathrm{E}-03 \\
(6.57)\end{array}$ & $\begin{array}{c}6.66 \mathrm{E}-03 \\
(6.96)\end{array}$ & $\begin{array}{c}4.21 \mathrm{E}-03 \\
(3.76)\end{array}$ & $\begin{array}{c}5.04 \mathrm{E}-03 \\
(4.59)\end{array}$ & $\begin{array}{c}4.78 \mathrm{E}-03 \\
(4.49)\end{array}$ & $\begin{array}{c}6.70 \mathrm{E}-03 \\
(6.26)\end{array}$ \\
\hline \multirow[t]{2}{*}{ Elevation } & $\begin{array}{c}-4.25 \mathrm{E}-04 \\
(-1.75)\end{array}$ & $\begin{array}{c}-3.96 \mathrm{E}-04 \\
(-1.74)\end{array}$ & $\begin{array}{c}-5.57 \mathrm{E}-04 \\
(-2.48)\end{array}$ & $\begin{array}{c}-5.49 \mathrm{E}-04 \\
(-2.1)\end{array}$ & $\begin{array}{c}-4.11 \mathrm{E}-04 \\
(-1.75)\end{array}$ & $\begin{array}{c}-4.30 \mathrm{E}-04 \\
(-1.83)\end{array}$ & Constant & $\begin{array}{c}1.21 \mathrm{E}+01 \\
(4.04)\end{array}$ & $\begin{array}{c}1.13 E+01 \\
(3.99)\end{array}$ & $\begin{array}{c}1.24 \mathrm{E}+01 \\
(4.25)\end{array}$ & $\begin{array}{c}1.33 \mathrm{E}+01 \\
(4.04)\end{array}$ & $\begin{array}{c}1.09 \mathrm{E}+01 \\
(3.64)\end{array}$ & $\begin{array}{c}1.07 \mathrm{E}+01 \\
(3.5)\end{array}$ \\
\hline & & & & & & & Adjusted R-squared & $8.47 \mathrm{E}-01$ & $8.45 \mathrm{E}-01$ & $8.42 \mathrm{E}-01$ & 8.44E-01 & $8.60 \mathrm{E}-01$ & 8.67E-01 \\
\hline
\end{tabular}

Notes: t-statistics in parenthesis. 